\title{
Gamma Ray Burst Optical Counterpart Search Experiment (GROCSE)
}

Hye-Sook Park ${ }^{1}$, Elden Ables ${ }^{1}$, Richard M. Bionta ${ }^{1}$, Linda Ott ${ }^{1}$, Eric Parker ${ }^{1}$, Carl Akerlof ${ }^{2}$, Brian Lee ${ }^{2}$, Scott Wallace ${ }^{2}$, Scott Barthelmy ${ }^{3}$, Paul Butterworth ${ }^{3}$, Thomas Cline ${ }^{3}$, Neil Gehrels ${ }^{3}$, Gerald Fishman ${ }^{4}$, Chryssa Kouveliotou ${ }^{5}$, Charles Meegan ${ }^{4}$, Donald Ferguson ${ }^{6}$

\author{
${ }^{1}$ Lawrence Livermore National Laboratory, Livermore, CA 94550 \\ ${ }^{2}$ University Of Michigan, Ann Arbor, MI 48109 \\ ${ }^{3}$ NASA/Goddard Space Flight Center, Greenbelt, MD 20771 \\ ${ }^{4}$ NASA/Marshall Spoce Flight Center, Huntsville, AL 35812
}

${ }^{5}$ USRA at NASA/Marshall Space Flight Center, Huntsville, AL 35812

${ }^{6}$ Earth Watch Inc., Pleasanton, CA 94550

GROCSE (Gamma-Ray Optical Counterpart Search Experiment) is a system of automated telescopes that search for simultaneous optical activity associated with gamma ray bursts in response to real-time burst notifications provided by the BATSE/BACODINE network. The first generation system, GROCSE $\mathrm{I}$, is sensitive down to $\mathrm{M}_{\mathrm{V}} \sim 8.5$ and requires an average of 12 seconds to obtain the first images of the gamma ray burst error box defined by the BACODINE trigger. The collaboration is now constructing a second generation system which has a 4 second slewing time and can reach $M_{\vee} \sim 14$ with a 5 second exposure. GROCSE II consists of 4 cameras on a single mount. Each camera views the night sky through a commercial Canon lens (f/1.8, focal length $200 \mathrm{~mm}$ ) and utilizes a $2 \mathrm{~K} \times 2 \mathrm{~K}$ Loral CCD. Light weight and low noise custom readout electronics were designed and fabricated for these CCDs. The total field of view of the 4 cameras is $17.6 \times 17.6^{\circ}$. GROCSE II will be operating by the end of 1995 . In this paper, we present an overview of the GROCSE system and the results of measurements with a GROCSE II prototype unit.

\section{INTRODUCTION}

As the origin of the gamma ray bursts (GRBs) is much debated, the existence or non-existence of any counterpart radiation at other wavelengths is recognized to be an important piece of the puzzle. In an attempt to place limits on the presence of optical radiation from GRBs in progress, we initiated a dedicated, rapid observation program in the summer of 1993. The

(C) 1996 American Institute of Physics 


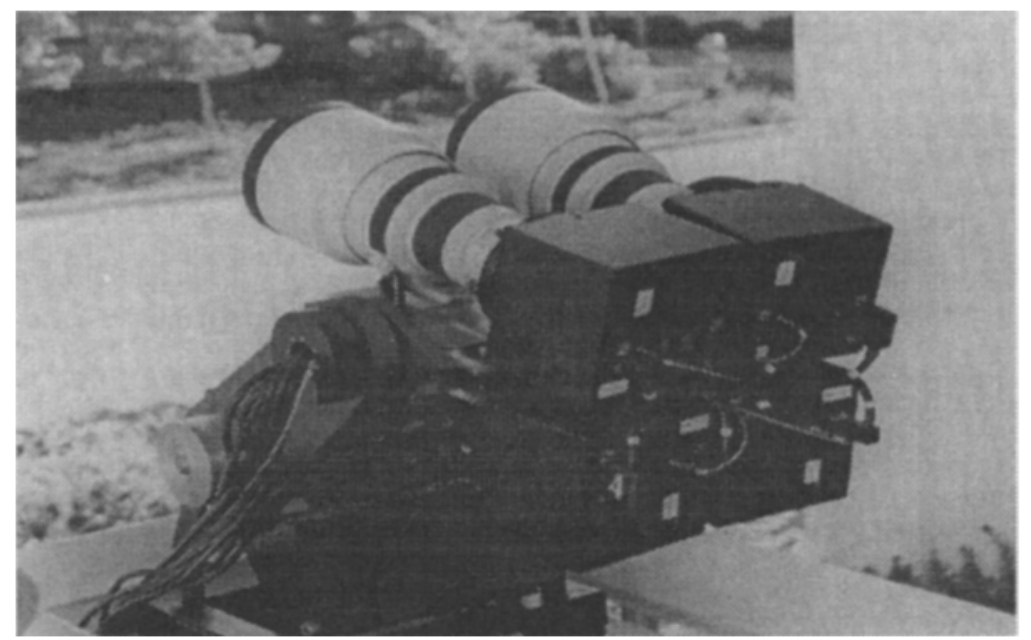

FIG. 1. GROCSE II System with 4 Cameras.

first generation experiment, GROCSE I (1) employed a wide-field-of-view telescope system at Lawrence Livermore National Laboratory. This system has a unique spherical lens and views a 60 degree field of view with 23 intensified CCD cameras. GROCSE I is mounted on a computer controlled Contraves mount that can point to any part of the sky in 10 to 15 seconds. The system was refurbished for the counterpart search and linked to BACODINE to receive GRB coordinates. Starting January 1994 we observed $>3600$ hours as of October 1995 and recorded 22 burst triggers. In several instances, the bursts lasted long enough for the GROCSE I system to slew over to its position and record imagery while the source was still emitting gamma ray radiation. However, we failed to identify any positive optical counterparts at the $M_{V} \sim 8.5$ sensitivity level amongst these events. Details on the GROCSE I experiment and results are given in (2).

Simple extrapolations of the gamma ray spectra to the optical-UV flux region predicts that the majority of the events will produce optical signals that are dimmer than $\mathrm{M}_{\mathrm{V}}>8.0$ (3). With the null results from GROCSE I experiment firmly established, we set out to construct a second generation system that achieves higher sensitivity and faster response time.

\section{GROCSE II}

The basic requirements for GRB counterpart searches utilizing the BATSE / BACODINE trigger are: 1) A field of view that is much wider than conventional telescope systems used in astronomy since the error box size for the BACODINE real-time GRB locations is up to $15^{\circ}$ in diameter; 2) A real time response to BACODINE triggers and fast slewing since the majority of GRBs last 1 1000 seconds. This requirement drives us toward compact and light 
weight camera and lens assemblies in order to keep the costs of the mount low. It also drives us to a fully automated system since robust automation is the only way of guaranteeing prompt observations at remote sites; 3) A short development time since the system has to start routine observations during the lifetime of the CGRO; 4) A low cost since "triggered" telescopes do not fall into conventional funding arenas normally supporting astronomy, astrophysics and space science. We optimized all these design specifications to produce the GROCSE II system.

The GROCSE II telescopes (Figure 1) utilize Canon's L series tele-photo lenses for the light gathering optics. These lenses have focal lengths of 200 $\mathrm{mm}$ at $\mathrm{f} / 1.8$ and, being commercially available, provide a good compromise between performance at low $f$ number and cost.

To obtain the widest field-of-view per camera we chose the largest area commercially available CCD imager, the Loral 442 which has a $2048 \times 2048$ pixel format with each pixel covering $15 \times 15 \mu \mathrm{m}^{2}$. Coupled with the Canon lens, each camera provides an $8.8 \times 8.8^{\circ}$ field of view. The complete GROCSE II system has 4 of these cameras to cover a $17.6 \times 17.6^{\circ}$ field of view. We developed custom readout electronics for these CCDs which consists of 3 boards: a CCD analog control board, a digitizer board, and a host computer interface board. The analog board controls the CCD clocking and video amplification which results in 20 electrons being digitized to one bit. The digitizer board digitizes the amplified video into 12 bits at $500 \mathrm{KHz}$. Then the digital pixel gray levels are serialized and sent to the camera host computer. The camera features a synchronous addressable serial interface (SASI) bus protocol to communicate with the host computer. The host computer can set the integration time, offset, shutter on/off, and camera enable. The measured readout noise for these cameras at room temperature is $\sim 50$ electrons.

The four cameras are mounted on an equatorial mount manufactured by Epoch Instruments (CA). This mount can point payloads of up to $50 \mathrm{lbs}$ to any part of sky within 4 seconds. As shown in the figure each camera is aligned $4.4^{\circ}$ off-axis to cover different parts of the $17.6 \times 17.6^{\circ}$ system field-of-view. The mount and the camera system will be housed inside a weather protective shell. In the clamshell's open position the cameras have an unobstructed view down to $20^{\circ}$ elevation angle.

Figure 2 shows a block diagram of the data acquisition system. Each camera has its own control computer (SUN/Sparc 2) with the camera interface built into a custom SBUS card. A SBUS dual ported memory buffers the image data which can be written either to the harddisk or directly to a 14 GByte $8 \mathrm{~mm}$ tape archival unit. The 4 camera computers are linked to a system host computer (SUN/Sparc 10) via ethernet. The host computer is connected to Internet through which it communicates to BACODINE. It also controls the mount, weather station, clam shell and UTC clock.

The on-line software is interrupt driven. The host exchanges packets with BACODINE once a minute and initiates various actions based on the packet type. When the host receives a packet announcing the coordinates of a gamma ray burst-in-progress it commands the mount to move to the burst coordinates 


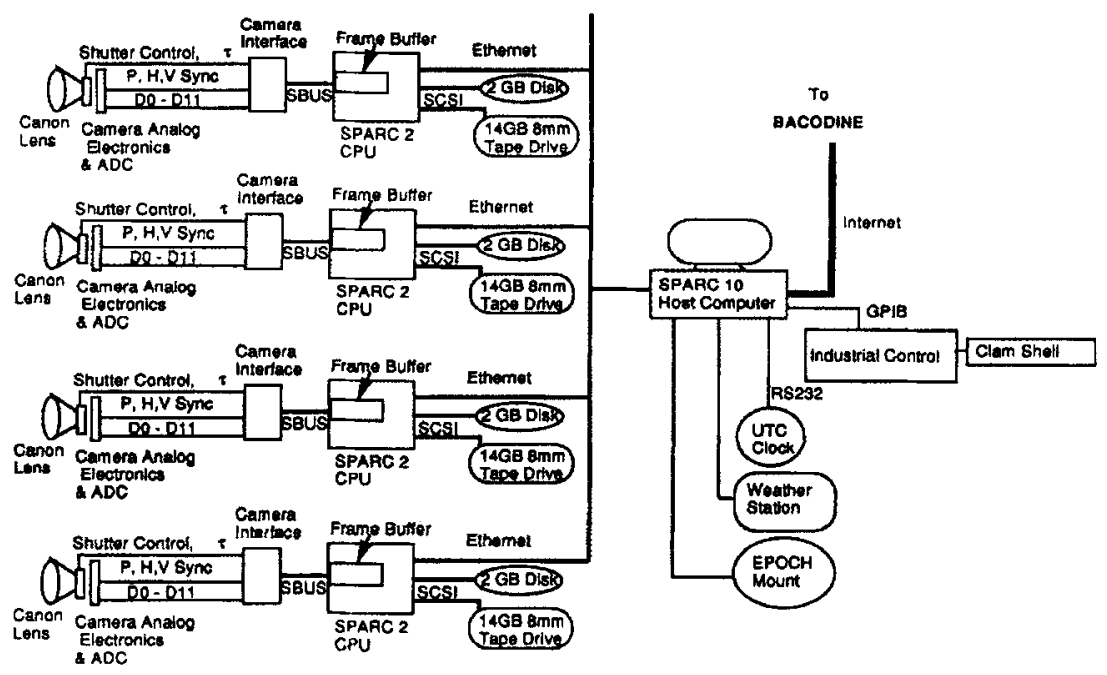

FIG. 2. GROCSE II Data Acquisition System.

then sends packets to the camera computers telling them to begin imaging. The host also continuously monitors the weather and will close the clamshell if rain or high winds are detected. The host fills idle time between bursts (which is most of the time) by commanding the system through a series of "sky patrols" designed to obtain archival images of the available night sky for comparison and diagnostic purposes.

\section{GROCSE II SYSTEM PERFORMANCE}

The expected performance of the GROCSE II system was estimated using both a radiometric model and outdoor sky images taken with a GROCSE II prototype camera. A $256 \times 256$ pixel portion $\left(1.1 \times 1.1^{\circ}\right)$ of GROCSE II prototype image field containing the star Vega is shown in Fig. 3. The FullWidth-Half-Maximum of the point spread function obtained by performing Gaussian fits to the stars in this field is 1.3 pixels. Based on the energy distribution of the point spread function and the measured signal/noise of these images, we expect that GROCSE II will achieve a limiting magnitude of $\mathrm{MV}_{\mathrm{V}} \sim 14$ at signal to noise ratio of 5.0 .

\section{SUMMARY}

GROCSE I is continuously running and producing GRB optical counterpart limits of $M_{V} \sim 8.5$. 


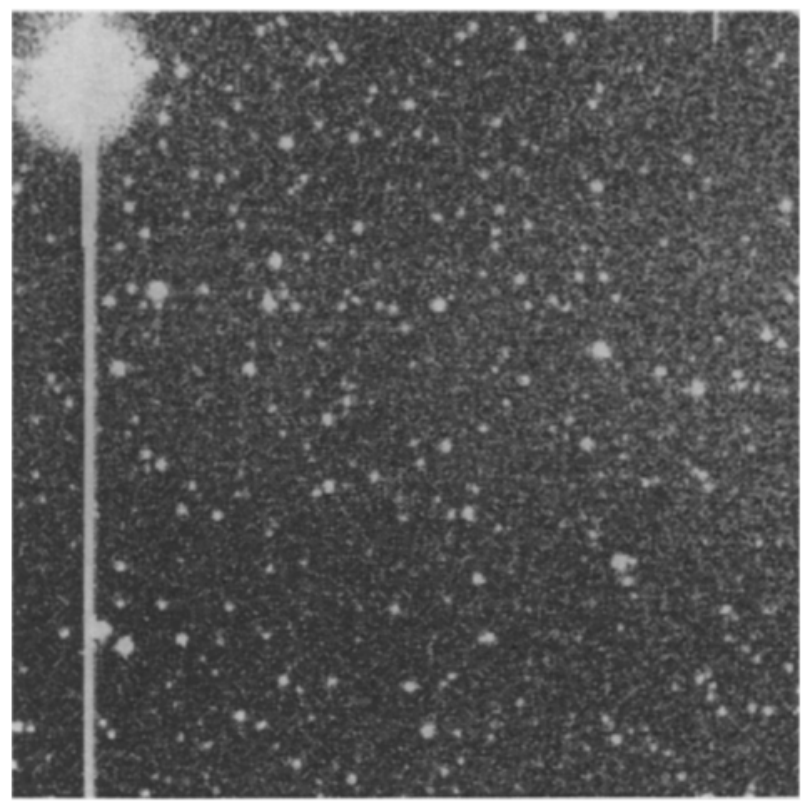

FIG. 3. A partial image taken with a GROCSE II prototype camera: $1.1 \times 1.1^{\circ}$ field near Vega.

The GROCSE II system will extend these limits to $M_{V}>14$ and will have faster response time. After a shakedown period scheduled for Dec. 1995, GROCSE II will be moved 15 miles east of Livermore, to an LLNL remote test facility that provides darker skies and less fog. We expect to start routine observations with GROCSE II at its remote site in early 1996.

\section{REFERENCES}

1. C. Akerlof, et al., in Gamma-Ray Bursts, eds. G.J. Fishman, J.J. Brainerd \& K. Hurley, AIP Conf. Proc. 307, 633 (AIP, New York, 1994).

2. B. Lee, et al., these proceedings (1996).

3. D. Band, L. Ford, Astrophys. \& Sp. Sci. 231 (1-2), 247 (1995). 